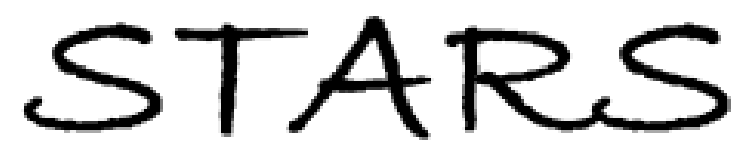

University of Central Florida

STARS

Faculty Bibliography 2000s

Faculty Bibliography

$1-1-2007$

\title{
Electron transport through single carbon nanotubes
}

G. Chai

H. Heinrich

University of Central Florida

L. Chow

University of Central Florida

T. Schenkel

Find similar works at: https://stars.library.ucf.edu/facultybib2000

University of Central Florida Libraries http://library.ucf.edu

This Article is brought to you for free and open access by the Faculty Bibliography at STARS. It has been accepted for inclusion in Faculty Bibliography 2000s by an authorized administrator of STARS. For more information, please contactSTARS@ucf.edu.

\section{Recommended Citation}

Chai, G.; Heinrich, H.; Chow, L.; and Schenkel, T., "Electron transport through single carbon nanotubes" (2007). Faculty Bibliography 2000s. 6920.

https://stars.library.ucf.edu/facultybib2000/6920

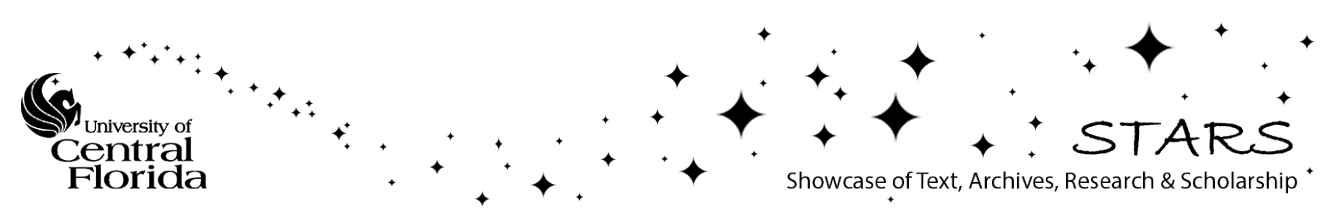




\section{Electron transport through single carbon nanotubes}

Cite as: Appl. Phys. Lett. 91, 103101 (2007); https://doi.org/10.1063/1.2778551

Submitted: 06 June 2007 . Accepted: 09 August 2007 . Published Online: 04 September 2007

G. Chai, H. Heinrich, L. Chow, and T. Schenkel

\section{ARTICLES YOU MAY BE INTERESTED IN}

Single- and multi-wall carbon nanotube field-effect transistors

Applied Physics Letters 73, 2447 (1998); https://doi.org/10.1063/1.122477

Self-Consistent Molecular-Orbital Methods. I. Use of Gaussian Expansions of Slater-Type Atomic Orbitals

The Journal of Chemical Physics 51, 2657 (1969); https://doi.org/10.1063/1.1672392

Ion beam modification of two-dimensional materials: Characterization, properties, and applications

Applied Physics Reviews 4, 011103 (2017); https://doi.org/10.1063/1.4977087

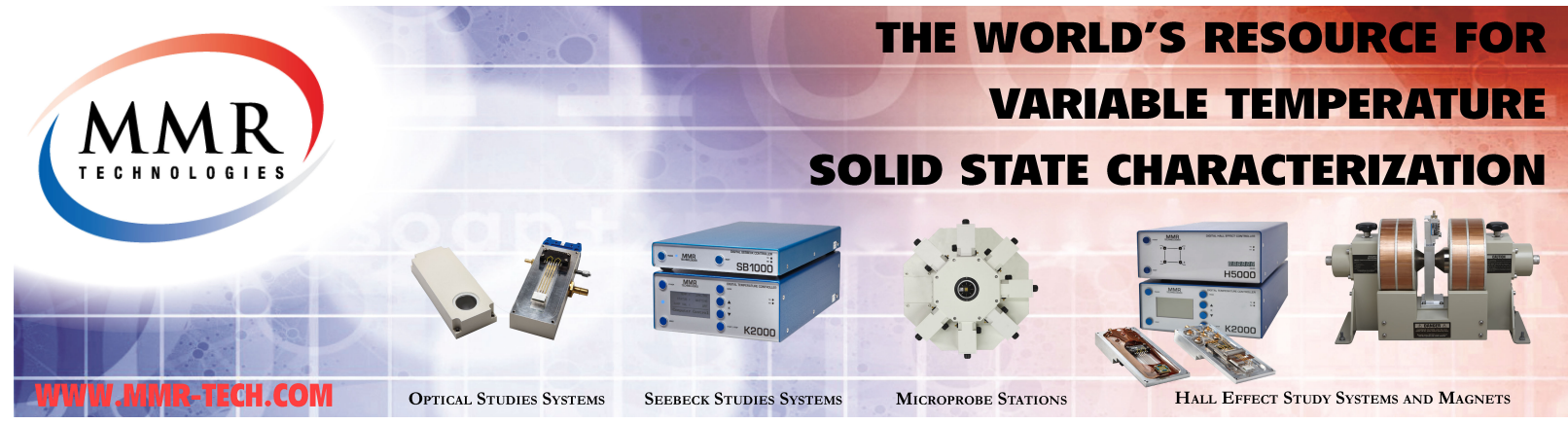




\title{
Electron transport through single carbon nanotubes
}

\author{
G. Chai \\ Apollo Technologies, Inc., 976 Florida Central Pkwy, Suite 112, Longwood, Florida 32750 \\ H. Heinrich and L. Chow ${ }^{\text {a) }}$ \\ Department of Physics, Advanced Materials Processing and Analysis Center, University of Central Florida, \\ Orlando, Florida 32816 \\ T. Schenkel \\ E. O. Lawrence Berkeley National Laboratory, Berkeley, California 94720
}

(Received 6 June 2007; accepted 9 August 2007; published online 4 September 2007)

\begin{abstract}
The authors report on the transport of energetic electrons through single, well aligned multiwall carbon nanotubes (CNTs). Embedding of CNTs in a protective carbon fiber coating enables the application of focused ion beam based sample preparation techniques for the nondestructive isolation and alignment of individual tubes. Aligned tubes with lengths of $0.7-3 \mu \mathrm{m}$ allow transport of $300 \mathrm{keV}$ electrons in a transmission electron microscope through their hollow cores at zero degree incident angles and for a misalignment of up to $1^{\circ}$. (C) 2007 American Institute of Physics.
\end{abstract}

[DOI: $10.1063 / 1.2778551]$

Control of the transport of atoms and molecules is at the heart of emerging nanotechnologies. Examples include delivery of specific molecules into living cells with nanometer precision, ${ }^{1}$ or the transport of large molecules through nanopores in sequencing applications. ${ }^{2}$ Carbon nanotubes have hollow cores with diameters ranging from one to tens of nanometers. The potential to use these narrow openings as transport channels for molecules ${ }^{3}$ and collimators or focusing elements for energetic particles has recently been recognized. ${ }^{4-7}$ To date, the fragility in handling of individual tubes has limited beam transport experiments to relatively short tubes with length of $15-60 \mathrm{~nm} .^{8}$ In this letter, we report on experiments with micrometer long tubes and energetic electrons in a transmission electron microscope (TEM). Electrons with kinetic energies of $300 \mathrm{keV}$ are found to efficiently transport through single, well aligned CNTs (carbon nanotubes). Alignment and controlled manipulation of single nanotubes is enabled by their encapsulation in a carbon fiber coating. ${ }^{9}$ This coating preserves and protects the tubes and it allows application of standard micromanipulation techniques to handle and align the otherwise fragile tubes.

The sample preparation process begins with the formation of the fiber protected CNT (F-CNT) with a recently developed chemical vapor deposition recipe. ${ }^{9}$ The resulting tubes are deposited on silicon substrates. Typical tubes are tens of micrometers long, with fiber coatings of about $1 \mu \mathrm{m}$ thickness surrounding the multiwall CNTs. The hollow cores have typical diameters of 5-20 nm. Following deposition and coating, a promising tube is selected in a focused ion beam (FIB) system, and standard lift-out techniquese ${ }^{10}$ are applied to isolate an encapsulated tube section. Figures 1(a)-1(c) show the stages of this lift-out process, where a selected F-CNT section is first coated with platinum, and trenches are then cut with the focused ion beam $(30 \mathrm{keV}$, $\mathrm{Ga}^{+}$) to release the sample and transfer it onto a copper grid for the electron transport experiments inside the TEM (we used an FEI Tecnai F30). In the TEM, we used a $300 \mathrm{keV}$

a) To whom correspondence should be addressed; electronic mail:
chow@ucf.edu electron beam with a divergence of $1 \mathrm{mrad}$ and a beam spot size of 3-4 $\mu \mathrm{m}$.

With an individual tube isolated and aligned in this way, the low divergence electron beam can now probe electron transmission through a single tube. Figure 2 shows a low magnification TEM image of the F-CNT on a silicon substrate where the F-CNT is mostly surrounded by the platinum deposit. The gap between the F-CNT and silicon substrate results from a shadowing effect during Pt deposition onto the F-CNT segment. The high magnification micrograph in Fig. 3(a) shows the electron transport through a single, well aligned F-CNT with a length of $0.72 \mu \mathrm{m}$. Tilting this sample to $5^{\circ}$ reveals the hollow core of the multiwall F-CNT with a diameter of about $13 \mathrm{~nm}$ [Fig. 3(b)]. The intensity profile from the line scan in Fig. 4(a) shows an electron beam spot corresponding to an open core width of $20 \mathrm{~nm}$ for a $3 \mu \mathrm{m}$ long F-CNT segment. When the F-CNT is tilted to $1^{\circ}$, as in Fig. 4(b), the intensity of transmitted electrons is reduced by about a factor of 5 and the beam profile becomes triangular. The opening angle of the F-CNT with $3 \mu \mathrm{m}$ length and an inner diameter of $20 \mathrm{~nm}$ (aspect ratio $\sim 150: 1$ ) is only $0.38^{\circ}$ and does thus not permit direct transmission at this incident angle. It is now interesting to consider the transport mechanisms that lead to the transmission

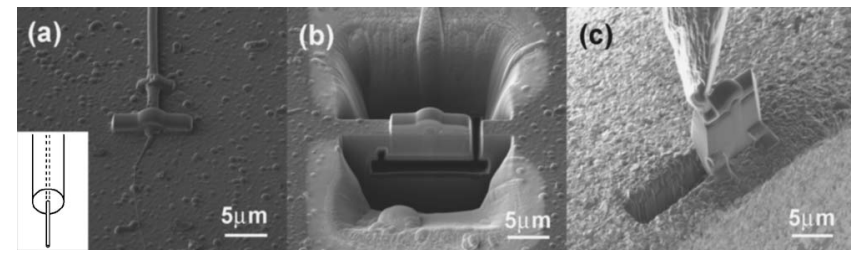

FIG. 1. (a) Scanning ion micrograph image of a fiber coated carbon nanotube. The tube section is coated with an additional layer of platinum in preparation of the lift-out process. A platinum layer is also used to attach the tube to the substrate to prevent buckling under ion beam exposure. The insert shows a schematic of the tube geometry. (b) Trenches are cut with the ion beam along a selected tube section to isolate the tube section for lift-out. (c) The isolated tube section is picked up with a micromanipulator and reattached to a TEM grid with thin platinum films formed by ion beam assisted deposition. 


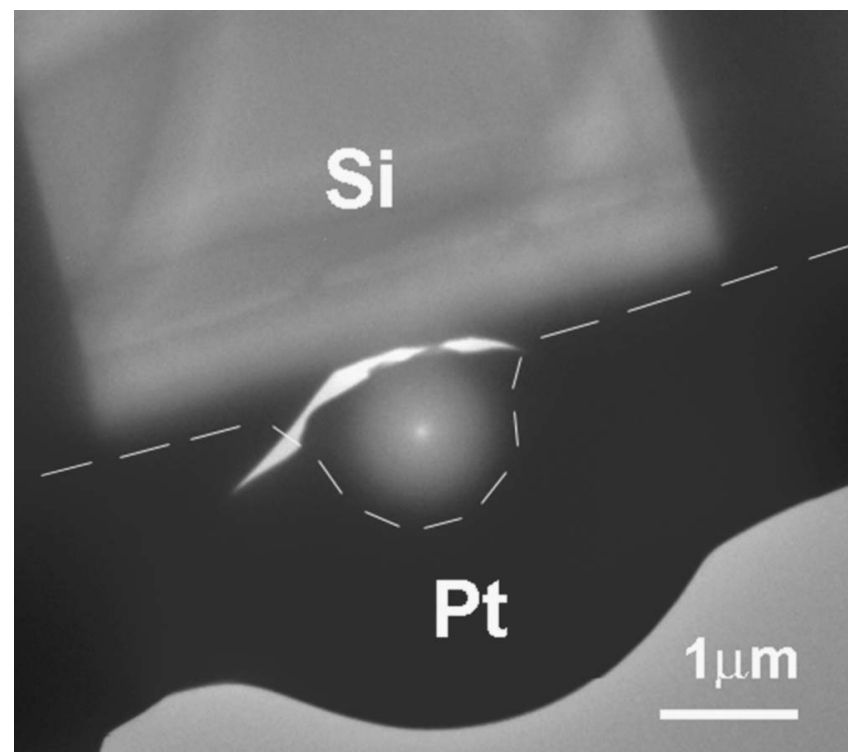

FIG. 2. Low magnification TEM micrographs of an isolated CNT section with a channel length of $0.72 \mu \mathrm{m}$ on a silicon substrate and with Pt deposit.

of electrons under these conditions. Recent studies of electron transport through $\mathrm{Al}_{2} \mathrm{O}_{3}$ capillaries with diameters of $140 \mathrm{~nm}$ and aspect ratios of 110:1 showed a guiding effect for $0.3 \mathrm{keV}$ electrons passing through insulating capillaries where the guiding originated from charge buildup on $\mathrm{Al}_{2} \mathrm{O}_{3}$ capillary walls. ${ }^{11}$ In the experiments described here, no such charge buildup is expected, since the multiwall CNTs are embedded in an amorphous carbon fiber coating that is not expected to trap charges efficiently. Also, no charging effects were observed in ion and electron beam imaging of F-CNT segments. An intriguing alternative mechanism is electron channeling ${ }^{12}$ along the carbon atom lattice and between the atomic layers surrounding the hollow core of the multiwall CNT. Elucidation and quantification of the detailed mechanisms that lead to the apparent guiding effect in the transport of energetic electrons through F-CNT segments will require more detailed studies of the angular dependence of transmission intensities. Studies of the angular dependence of electron transmission were hampered in our experiments by the tube closing after exposure to the electron beam for several minutes. The origin of this closure is believed to be the electron beam assisted deposition of carbon, where the source of the carbon is likely to be the protective coating that surrounds and protects the tube. We found that tubes can be reopened and electron beam transmission can be restored by ion beam sputter cleaning of the tube opening in a FIB, and future experiments will be conducted in a dual beam FIB for in situ preparation and probing of ion and electron transmis-

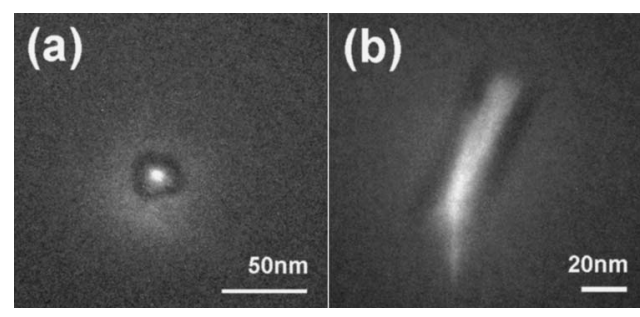

FIG. 3. TEM images of a single CNT section aligned to the electron beam under $0^{\circ}$ tilt (a) and under a tilt angle of $5^{\circ}$ (b). The image with large tilt angle reveals the inner diameter of the tube of $13 \mathrm{~nm}$.

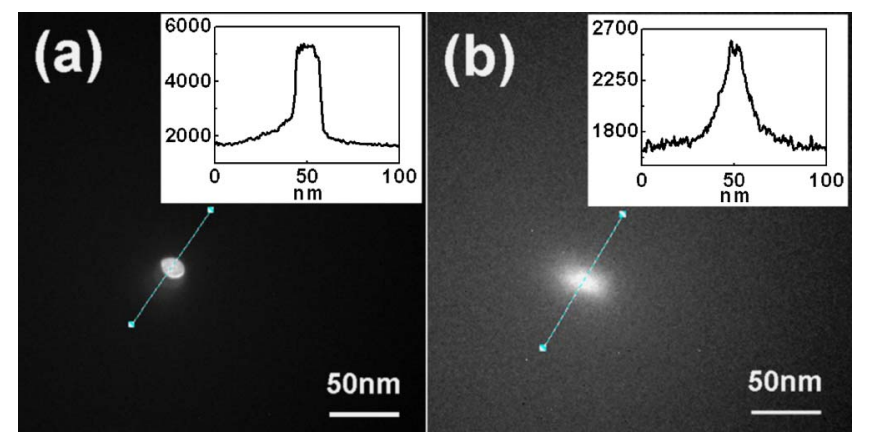

FIG. 4. (Color online) TEM micrographs of a $3 \mu \mathrm{m}$ long CNT section under a tilt of $0^{\circ}$ (a) and under a tilt of $1^{\circ}$ (b). The inserts show the intensity profiles of the transmitted electron beam in the imaging plane along the indicated line scans.

sions through single, aligned tubes. Electron transmission was observed for multiwall tubes with lengths up to $3 \mu \mathrm{m}$, and future work aims at exploring electron transmission through longer tubes and single walled tubes.

We also observed evidence for a Fresnel diffraction effect ${ }^{8}$ in electron transport through a single tube. Here, the electron beam impinged on the aligned tube sample in a condition of underfocusing by $2 \mu \mathrm{m}$ from the Gaussian focus condition. Figure 5 shows the TEM micrograph with a line scan of the intensity profile in the insert. In this condition, the electron beam is focused by transmission through the F-CNT channel to a spot with a diameter of only $6 \mathrm{~nm}$.

The demonstration of efficient electron transport through single, micrometer long, and well aligned carbon nanotubes has the potential to realize new classes of collimators and beam optics for energetic particles-ions ${ }^{13}$ as well as electrons. CNT beam transport elements can, e.g., enable the placement of dopant atoms with nanometer precision, beyond the current limits in ion beam focusing. ${ }^{13}$ The use of fiber coated carbon nanotubes makes the handling of single tubes robust and compatible with standard micromanipulation techniques, and testing that a tube is really open with widely available TEMs might enable transmission and material transport experiments through CNTs with much higher rates of reproducibility than previously possible.

One of the authors (L.C.) acknowledges partial financial support from Apollo Technologies, Inc. and Florida High

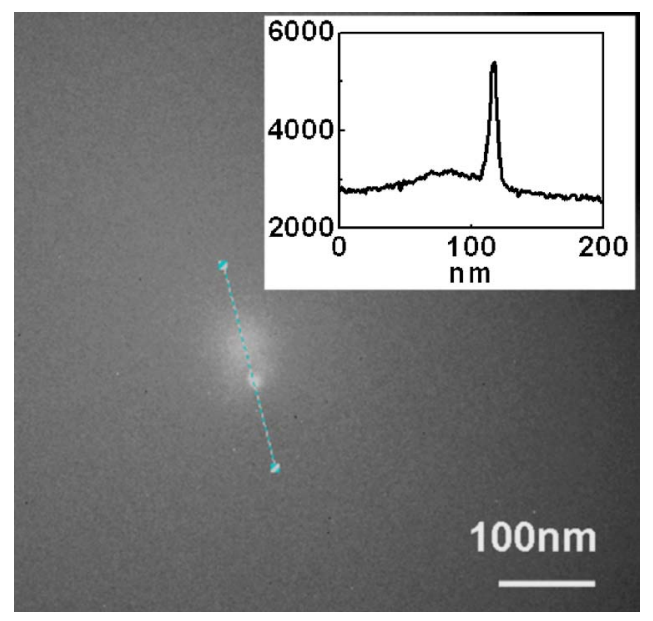

FIG. 5. (Color online) TEM micrograph for the $3 \mu \mathrm{m}$ long CNT section under $0^{\circ}$ recorded with the electron beam underfocused by $2 \mu \mathrm{m}$. 
Tech Corridor Research Program. Work at LBNL was supported by the National Security Agency under Contract No. MOD 713106A, and by the Department of Energy under Contract No. DE-AC02-05CH11231.

${ }^{1}$ X. Chen, A. Kis, A. Zettl, and C. R. Bertozzi, Proc. Natl. Acad. Sci. U.S.A. 104, 8218 (2007).

${ }^{2}$ M. Rhee and M. A. Burns, Trends Biotechnol. 25, 174 (2007).

${ }^{3}$ Y. Maniwa, K. Matsuda, H. Kyakuno, S. Ogasawara, T. Hibi, H. Kadowaki, S. Suzuki, Y. Achiba, and H. Kataura, Nat. Mater. 6, 135 (2007).

${ }^{4}$ A. V. Krasheninnikov and K. Nordlund, Phys. Rev. B 71, 245408 (2005). ${ }^{5}$ D. P. Zhou, Y. H. Song, Y. N. Wang, and Z. L. Miskovic, Phys. Rev. A 73, 033202 (2006).
${ }^{6}$ S. Bellucci, V. M. Biryukov, Y. A. Chesnokov, V. Guidi, and W. Scandale, Nucl. Instrum. Methods Phys. Res. B 202, 236 (2003).

${ }^{7}$ C. S. Moura and L. J. Amaral, J. Phys. Chem. B 109, 13515 (2005).

${ }^{8}$ A. Kruger, M. Ozawa, and F. Banhart, Appl. Phys. Lett. 83, 5056 (2003). ${ }^{9}$ S. Kleckley, G. Chai, D. Zhou, R. Vanfleect, and L. Chow, Carbon 41, 833 (2003).

${ }^{10}$ M. H. F. Overwijk, F. C. Vandenheuvel, and C. W. T. Bullelieuwma, J. Vac. Sci. Technol. B 11, 2021 (1993).

${ }^{11}$ A. R. Milosavljević, Gy. Víkor, Z. D. Pešić, P. Kolarž, D. Šević, B. P. Marinković, S. Mátéfi-Tempfli, M. Mátéfi-Tempfli, and L. Piraux, Phys. Rev. A 75, 030901(R) (2007).

${ }^{12}$ D. S. Gemmel, Rev. Mod. Phys. 46, 129 (1974).

${ }^{13}$ A. Persaud, S. J. Park, J. A. Liddle, I. W. Rangelow, J. Bokor, and T. Schenkel, Nano Lett. 5, 1087 (2005). 